\title{
Top-quark properties and mass measurements with the ATLAS detector
}

\author{
Tomas Dado ${ }^{1,2, a}$ \\ On behalf of the ATLAS collaboration \\ ${ }^{1}$ Comenius University in Bratislava \\ ${ }^{2}$ Georg-August-Universität Göttingen
}

\begin{abstract}
The top quark is unique among the known quarks in that it decays before it has an opportunity to form hadronic bound states. This makes measurements of its properties particularly interesting as one can access directly the properties of a bare quark. The latest measurements of these properties with the ATLAS detector at the LHC [1] are presented. Measurements of top-quark spin observables in top-antitop events, each sensitive to a different coefficient of the spin density matrix, are presented and compared to the Standard Model predictions. The helicity of the $W$ boson from the top decays and the production angles of the top quark are further discussed. Limits on the rate of flavour changing neutral currents in the production or decay of the top quark are reported. The production of top-quark pairs in association with $W$ and $Z$ bosons is also presented. The measurement probes the coupling between the top quark and the $Z$ boson. The cross-section measurement of photons produced in association with top-quark pairs is also discussed. These process are all compared to the best available theoretical calculations. The latest ATLAS measurements of the top-quark mass in lepton+jets, dilepton, and all-hadronic final states are also reported. In addition, measurements aiming to measure the mass in a well-defined scheme are presented.
\end{abstract}

\section{Introduction}

With the mass around $173 \mathrm{GeV}$, the top quark is the heaviest known elementary particle. Due to its large mass, the top quark in the Standard Model (SM) has large decay width corresponding to very short mean life-time of around $0.5 \times 10^{-24} \mathrm{~s}$. Because of this short mean life-time, the top quark decays before it can form a bound state and transfers all its properties to its decay products. This makes the top quark unique among other quarks, as it allows to study properties of a bare quark. Moreover, the top quark Yukawa coupling to Higgs boson is $O(1)$ which implies that the top quark could play a special role in the process of electroweak symmetry breaking. Many Beyond Standard Model (BSM) scenarios predict different values for some of the properties of the top quark. Thus precisely measuring the properties of the top quark can test SM predictions and constrain some of the BSM parameters. In addition, the top quark is produced at very short distances - a characteristic strong coupling constant $\alpha_{\mathrm{s}}$ is of the order of 0.1 . This makes top quark a perfect object to study perturbative QCD.

\footnotetext{
a e-mail: tomas.dado@cern.ch
} 
Currently, the Large Hadron Collider (LHC) is referred as "a top factory", since the top quarks are produced abundantly during collisions. This makes the LHC an ideal place to measure the topquark properties. Top quarks are predominantly produced in pairs in the LHC, however single-top production is also possible via electroweak production mechanism. In the SM the top quark decays almost exclusively into $W$ boson and $b$-quark. Depending on the subsequent decay of the $W$ boson there are three decay channels of top-quark pair: all-hadronic - both $W$ bosons decay into pair of quarks, semi-leptonic ${ }^{\star}$ - one $W$ boson decays into pair of quarks and the other $W$ boson decays into charged lepton and its corresponding neutrino and finally dileptonic - both $W$ bosons decay into charged leptons and their corresponding neutrino.

\section{Measurement of top-quark spin observables}

Top quarks are assumed to be produced unpolarised, which means their spins are not aligned with any direction. However, spins of top and antitop quark from pair production are correlated. The level of correlation depends on reference quantisation axis and the production process. Top quarks decay before any spin-flip occurs, thus the full information about the quark spin is transferred to its decay products.

The most recent ATLAS result [2] analyses fifteen observables sensitive to top-quark pairs' spin density matrix. The measurement uses $t \bar{t}$ dileptonic data at centre-of-mass energy $\sqrt{s}=8 \mathrm{TeV}$. Neutrino weighting $[3,4]$ technique is used to fully reconstruct $t \bar{t}$ kinematics. Normalised doubledifferential cross-section for $t \bar{t}$ production and decay is of the form

$$
\frac{1}{\sigma} \frac{d^{2} \sigma}{d \cos \theta_{+}^{a} d \cos \theta_{-}^{b}}=\frac{1}{4}\left(1+B_{+}^{a} \cos \left(\theta_{+}^{a}\right) B_{-}^{b} \cos \theta_{-}^{b}-C(a, b,) \cos \left(\theta_{+}^{a}\right) \cos \theta_{-}^{b}\right)
$$

where $B^{a}, B^{b}$ and $C(a, b)$ are the polarisation and spin correlations along the spin quantisation axes, $a$ and $b$. Angles $\theta^{a}$ and $\theta^{b}$ are defined as angles between the momentum direction of a top-quark decay particle in its parent top-quark's rest frame and the axis $a$ or $b$ respectively. Three different quantization axes are compared: helicity axis $(k)$ - defined as the top-quark direction in the $t \bar{t}$ rest frame, transverse axis $(n)$ - defined to be transverse to the production plane created by top-quark direction and the beam axis and $r$-axis $(r)$ - axis orthogonal to the other two axes. Detector level distributions are unfolded to parton level, shown in Figure 1 and compared to theory predictions. Results are also unfolded to stable particle level and are compared to prediction from MC simulation (Powheg-hvq+Pythia6). At parton level, the measurements along the helicity axes are

$$
\begin{array}{rlr}
B_{+}^{k} & = & -0.044 \pm 0.038[ \pm 0.027 \text { (mass) }], \\
B_{-}^{k} & = & -0.064 \pm 0.040[ \pm 0.027 \text { (mass) }], \\
C(k, k) & = & 0.296 \pm 0.093[ \pm 0.037 \text { (mass) }],
\end{array}
$$

where $+(-)$ denotes decay products of top (anti-top) quark. Systematic uncertainties are dominated by uncertainties in signal modelling. The results are in good agreement with NLO SM predictions. The measured spin correlation along the transverse axis differs from zero correlation with a significance of $5.1 \sigma$. Ten observables are measured for the first time and all distributions are in agreement with SM predictions.

\footnotetext{
^Sometimes referred to as "lepton+jets".
} 


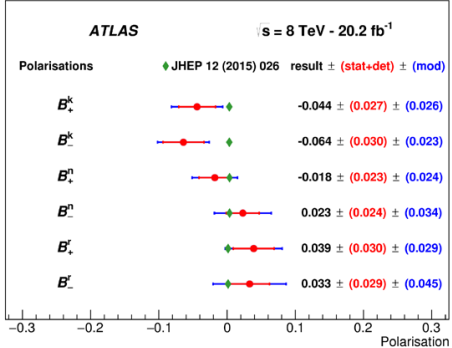

(a)

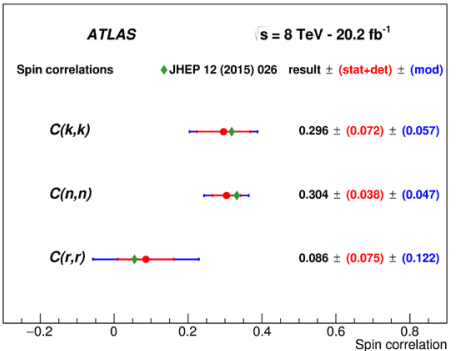

(b)

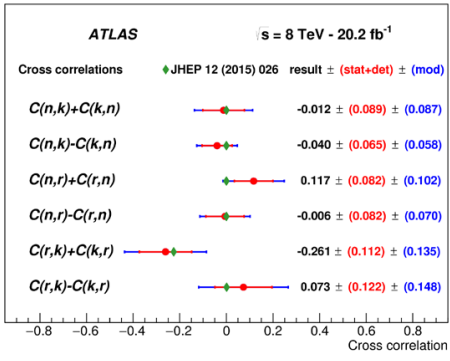

(c)

Figure 1. Comparison of the measured polarisations (a), spin correlations (data points) (b) and crosscorrelations (c) with predictions from the SM (diamonds) for the parton-level measurement. Inner bars indicate uncertainties obtained from the marginalisation, outer bars indicate modelling systematics, summed in quadrature. The widths of the diamonds are chosen for illustrative purposes only [2].

\section{$3 W$ helicity measurement in top-quark events}

In SM, top quark decays almost exclusively to $W$ boson and $b$ quark. The $W t b$ decay vertex has $V-A$ structure, where $V$ and $A$ refer to the vector and axial vector components of the weak coupling. Due to this structure and large difference between top quark and $b$ quark mass, the $W$ boson from top quark decay has only left-handed and longitudinal polarisation, the right-handed polarisation is heavily suppressed.

The $W$ helicity in semileptonic decays of top-quark pair is measured with the ATLAS detector at centre-of-mass energy $\sqrt{s}=8 \mathrm{TeV}$ [5]. An observable sensitive to the $W$ boson polarisation is angle $\theta^{*}$ - angle between $b$-quark from the top-quark decay and a direct $W$ boson decay product in the $W$ boson rest frame. Polarisations of leptonically and hadronically decaying $W$ bosons are analysed. Charged lepton and down-type quark are used as direct decay products of $W$ bosons in leptonically and hadronically decaying channels respectively. Extended KLFitter [7] technique is used to fully reconstruct $t \bar{t}$ kinematics. The extension enables separation between up- and down-type quark from the $W$ decay. Pure helicity templates of $\cos \theta^{*}$ distributions are fitted to data. The measured $W$ helicities as well as theoretical NNLO predictions [8] are summarized in Table 1. Dominant systematic uncertainties arise from uncertainties in jet energy reconstruction and signal modelling. No significant deviation from SM prediction is observed, thus the limits on possible anomalous couplings in Effective Field Theory, e.g. (EFT) [6], are calculated and presented in Figure 2. This is the most precise $W$ helicity measurement till date.

Table 1. Measured helicity fractions for longitudinal $\left(F_{0}\right)$, left-handed $\left(F_{L}\right)$ and right-handed $\left(F_{R}\right)$ polarisations from leptonically and hadronically decaying $W$ boson. The total uncertainty is shown. Theory predictions

(NNLO) are presented.

\begin{tabular}{llll}
\hline Polarisation & Theory & Leptonic & Hadronic \\
\hline$F_{0}$ & $0.687 \pm 0.005$ & $0.709 \pm 0.019$ & $0.659 \pm 0.053$ \\
$F_{L}$ & $0.311 \pm 0.005$ & $0.299 \pm 0.015$ & $0.281 \pm 0.066$ \\
$F_{R}$ & $0.0017 \pm 0.0001$ & $-0.008 \pm 0.014$ & $0.061 \pm 0.103$ \\
\hline
\end{tabular}




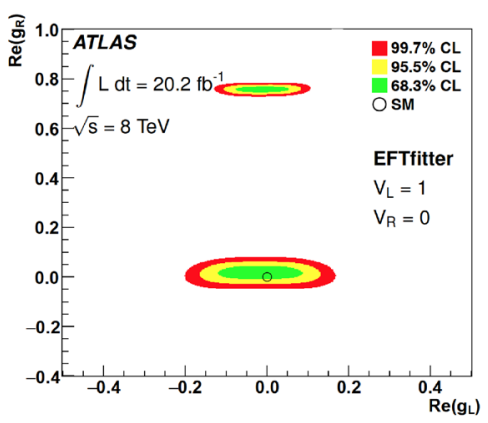

(a)

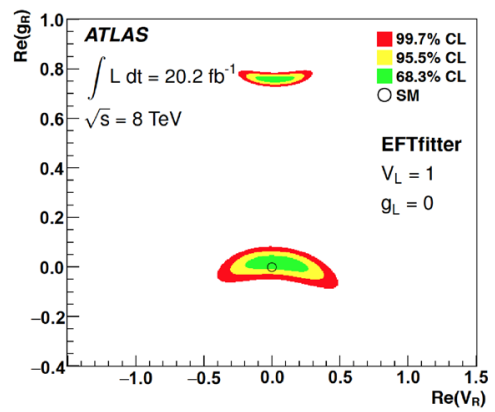

(b)

Figure 2. (a) Limits on the anomalous left- and right-handed tensor couplings of the Wtb decay vertex as obtained from the measured $W$ boson helicity fractions from the leptonic analyser. (b) Limits on the right-handed vector and tensor coupling. As the couplings are assumed to be real, the real part corresponds to the magnitude. Unconsidered couplings are fixed to their SM values [5].

\section{Direct measurement of the top-quark decay width}

Decay width is an important property of every elementary particle. In SM, the decay width depends on the particle mass. NNLO prediction [9] yields top-quark decay width $\Gamma_{t}=1.32 \mathrm{GeV}$ for the top-quark mass $m_{t}=172.5 \mathrm{GeV}$.

The first direct measurement with the ATLAS detector [10] uses $20.2 \mathrm{fb}^{-1}$ of data at centre-of-mass energy $\sqrt{s}=8 \mathrm{TeV}$. The measurement focuses on semileptonic decay of $t \bar{t}$. Two variables sensitive to decay width of top quark are used: $m_{\ell b}$ - invariant mass of lepton and corresponding $b$-jet from top-quark decay and $\Delta R_{\min }\left(j_{b}, j_{\text {light }}\right)-\Delta R^{\dagger}$ between $b$-jet from hadronically decaying top and closest light jet from hadronically decaying $W$ boson. Events are split by lepton flavour $(e$ or $\mu$ ), number of $b$-tagged jets (exactly one $b$-tagged jet and at least two $b$-tagged jets) and jet $|\eta|=1$ to decrease systematic uncertainties in jet energy reconstruction. Templates with different widths are fitted to data and the measured width is

$$
\Gamma_{\text {top }}=1.76 \pm 0.33 \text { (stat.) }{ }_{-0.68}^{+0.79} \text { (syst.) } \mathrm{GeV} \text {, }
$$

assuming top mass $m_{t}=172.5 \mathrm{GeV}$. Measurement is limited by jet energy reconstruction and signal modelling uncertainties. The measured width is in good agreement with the SM prediction.

\section{Searches for FCNC with top quarks}

Flavour-changing neutral currents (FCNCs) are forbidden at tree level in the SM and are heavily suppressed by the GIM mechanism. However, different BSM scenarios predict large effective couplings which are orders of magnitude larger than those in the SM. Searches for FCNC can give either a hint or constrain some of the BSM scenarios.

\footnotetext{
${ }^{\dagger} \Delta R \equiv \sqrt{(\Delta \eta)^{2}+(\Delta \phi)^{2}}$.
} 


\subsection{Search for $t \rightarrow q H(H \rightarrow \gamma \gamma)$}

The ATLAS collaboration performed a search [11] for $t \rightarrow q H(\rightarrow \gamma \gamma)$, where $q=c, u$; at the centreof-mass energy $\sqrt{s}=13 \mathrm{TeV}$, corresponding to $36.1 \mathrm{fb}^{-1}$. In $\mathrm{SM}$, this process is heavily suppressed with branching ratio of approximately $3 \times 10^{-15}$. Two event selections are applied: hadronic selection where the non-FCNC top quark decays hadronically $t \rightarrow b W(W \rightarrow j j)$ and leptonic selection where $t \rightarrow b W(W \rightarrow \ell v)$. Events are required to have diphoton mass $100<m_{\gamma \gamma}<160 \mathrm{GeV}$. Events are further split into two complementary categories. In the first category events passing all selection criteria for hadronic or leptonic selection are considered. In the second category events passing all selection criteria except the criterion on reconstructed top-quark mass $120<m_{j j j}<220 \mathrm{GeV}$ for hadronic selection and $130<m_{j \ell v}<210 \mathrm{GeV}$ for leptonic selection are considered. Control regions are used to constrain dominant backgrounds: associated production of top-quark pair with a vector boson and diboson processes. No significant excess of events over expectation is observed. Limits on FCNC branching ratios are set

$$
\mathrm{BR}(t \rightarrow c H)<2.2 \times 10^{-3}, \quad \mathrm{BR}(t \rightarrow u H)<2.4 \times 10^{-3} \quad \text { at } 95 \% \mathrm{CL} .
$$

This corresponds to limits on anomalous couplings

$$
\sqrt{\lambda_{t c H}^{2}+\lambda_{t u H}^{2}}<0.090 \text { at } 95 \% \mathrm{CL}
$$

assuming $m_{H}=125.09 \mathrm{GeV}$. Dominant systematic uncertainties arise from jet energy scale reconstruction and signal modelling. Current sensitivity allows for a probe in regions of flavour violating Yukawa coupling in Two Higgs Doublet Models (2HDM).

\subsection{Search for $t \rightarrow q Z(Z \rightarrow \ell \ell)$}

A search for $t \rightarrow q Z(Z \rightarrow \ell \ell)$ is performed by the ATLAS collaboration using centre-of-mass $\sqrt{s}=$ $8 \mathrm{TeV}$ data, corresponding to $20.3 \mathrm{fb}^{-1}$ [12]. Branching ratio predicted by SM for this process is of the order of $10^{-14}$. The search focuses on top-quark pair events with $Z$ boson decaying leptonically $(Z \rightarrow \ell \ell)$ and the non-FCNC top quark decaying semileptonically $t \rightarrow b W(W \rightarrow \ell v)$. Kinematics of $t \bar{t}$ system is reconstructed using $\chi^{2}$ kinematic method. Additionally, $\chi^{2}<6$ is required to improve the purity of the selection. No significant deviation from SM prediction is observed and the limits on $t \rightarrow q Z$ are set as

$$
\mathrm{BR}(t \rightarrow q Z)<7 \times 10^{-4} \text { at } 95 \% \mathrm{CL} \quad\left(8_{-2}^{+4} \times 10^{-4} \text { expected }\right) .
$$

Uncertainty in the background modelling is the dominant systematic uncertainty of this measurement. Combination with other FCNC searchers is shown in Figure 3.

\subsection{Search for $q g \rightarrow t$}

Searching for FCNC decays of top quark to an up-type quark and gluon $(t \rightarrow q g)$ is extremely difficult due to overwhelming QCD background. Alternatively, one may look for FCNC production of single top quark. This search is performed by the ATLAS collaboration [13] using centre-of-mass energy $\sqrt{s}=8 \mathrm{TeV}$, corresponding to $20.3 \mathrm{fb}^{-1}$. Single top quark produced via FCNC has different kinematic 


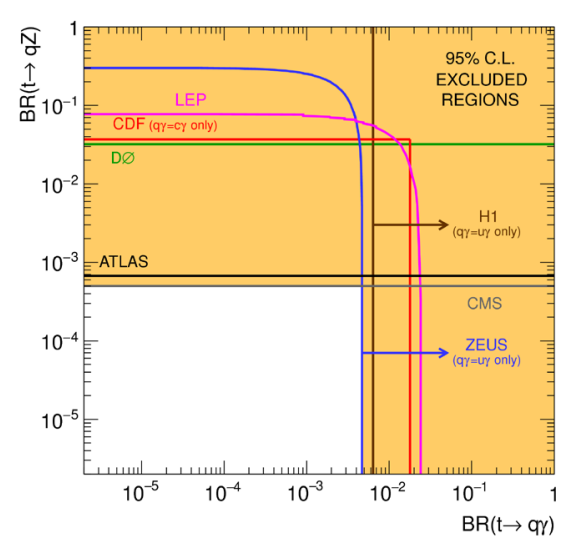

(a)

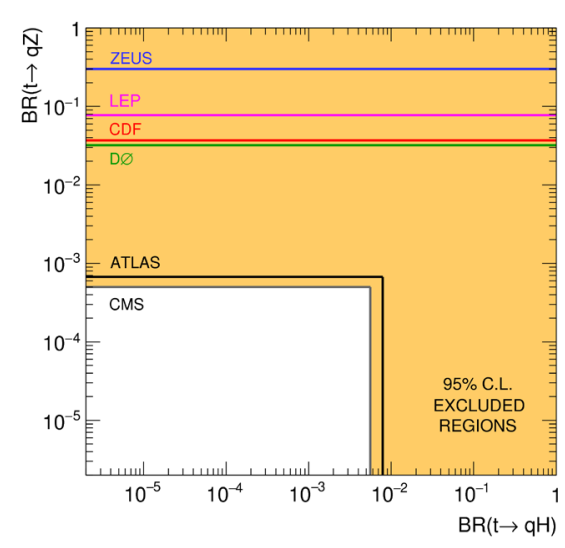

(b)

Figure 3. The current $95 \% \mathrm{CL}$ observed limits on the (a) $\mathrm{BR}(t \rightarrow q \gamma)$ vs $\mathrm{BR}(t \rightarrow q Z)$ and (b) $\mathrm{BR}(t \rightarrow$ $q H)$ vs $\mathrm{BR}(t \rightarrow q Z)$ planes are shown. The ATLAS lines correspond to the limit on $\mathrm{BR}(t \rightarrow q Z)$ set in this measurement [12].

properties: FCNC single top-quark $p_{\mathrm{T}}$ distribution is softer compared to single top SM prediction; the $W$ boson from the top-quark decay has high momentum and its decay products tend to have small angles and the top-quark charge asymmetry differs between FCNC processes and SM processes in the ugt channel. Unfortunately, none of the observables have enough separation power to distinguish top quarks produced via FCNC. Thus artificial neural network (NN) is used. NN response is fitted to observed data. Control region is used to constrain the dominant $W+$ jets background. No significant excess over SM prediction is observed and limit on production cross section is set as

$$
\sigma_{q g \rightarrow t} \times \mathcal{B}(t \rightarrow W b)<3.4 \mathrm{pb} \quad \text { at } 95 \% \mathrm{CL} .
$$

The limit on production cross section is translated to limit on coupling constant divided by scale factor of new physics $(\Lambda)$ and on branching fractions $(\mathcal{B})$

$$
\begin{array}{ll}
K_{\text {ugt }} / \Lambda<5.8 \times 10^{-3} \mathrm{TeV}^{-1}, & K_{\text {cgt }} / \Lambda<13 \times 10^{-3} \mathrm{TeV}^{-1}, \\
\mathcal{B}(t \rightarrow u g)<4.0 \times 10^{-5}, & \mathcal{B}(t \rightarrow c g)<20 \times 10^{-5} .
\end{array}
$$

Figure 4 illustrates upper limits on coupling constants and on branching ratios. These are the most stringent limits till date. Summary of recent FCNC searches performed by ATLAS and CMS is presented in Figure 5.

\section{Associated production of $t \bar{t}$ and vector boson}

Associated production of $t \bar{t}$ and $Z$ boson can probe neutral-current coupling of top quark and $Z$ boson. The production cross section is also sensitive to the third component of the top-quark weak isospin and to possible anomalous couplings from BSM. The ATLAS collaboration measures $t \bar{t} Z$ and $t \bar{t} W$ 


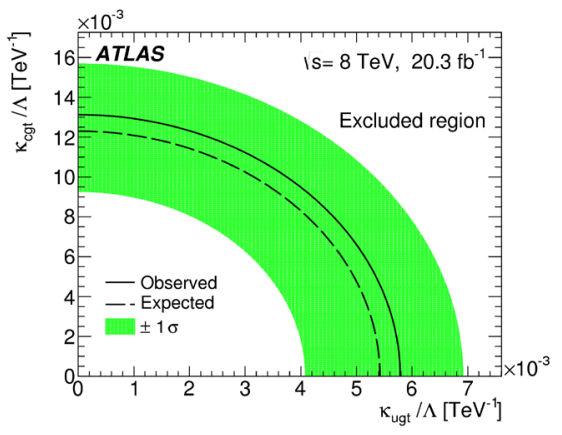

(a)

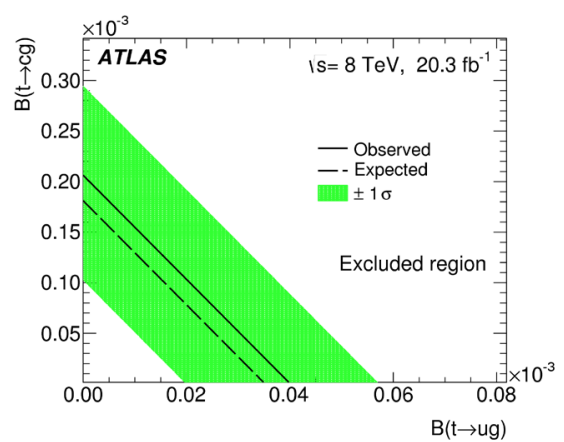

(b)

Figure 4. (a) Upper limit on the coupling constants $K_{u g t}$ and $K_{c g t}$ and (b) on the branching fractions $\mathrm{B}(t \rightarrow u g)$ and $\mathrm{B}(t \rightarrow c g)$. The shaded band shows the one standard deviation variation of the expected limit [13].

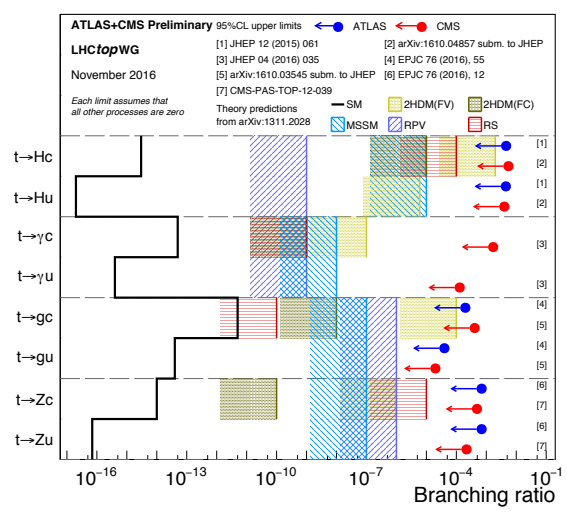

Figure 5. Summary of the current $95 \%$ confidence level observed limits on the branching ratios of the top-quark decays via flavour changing neutral currents to a quark and a neutral boson $t \rightarrow X q(X=g, Z, \gamma$ or $H ; q=u$ or $c)$ by the ATLAS and CMS Collaborations compared to several new physics models [14].

cross sections from data collected in 2015 using centre-of-mass energy $\sqrt{s}=13 \mathrm{TeV}$, corresponding to integrated luminosity of $3.2 \mathrm{fb}^{-1}$ [15]. The measurement focuses on three different signal regions: same-sign di-muon channel - targeting $t \bar{t} W$ production; trilepton channel - targeting both $t \bar{t} W$ and $t \bar{t} Z$ production and finally tetralepton channel - targeting $t \bar{t} Z$ production. Each analysis channel is further divided into multiple regions in order to enhance the sensitivity to signal. In total, nine signal and two control regions enter the profile likelihood fit in order to extract the cross sections for $t \bar{t} W$ and $t \bar{t} Z$ production. Only semileptonic and dileptonic decay channels of top-quark pair are considered. Observed cross-sections are

$$
\begin{aligned}
\sigma_{t \bar{Z} Z} & =0.92 \pm 0.29 \text { (stat.) } \pm 0.10 \text { (syst.) pb, } \\
\sigma_{t \bar{t} W} & =1.50 \pm 0.72 \text { (stat.) } \pm 0.30 \text { (syst.) pb }
\end{aligned}
$$


with the observed significance of $3.9 \sigma$ and $2.2 \sigma$ with respect to background-only hypothesis for $t \bar{t} Z$ and $t \bar{t} W$ respectively. The correlation between the observed cross sections is $13 \%$. Figure 6 shows observed cross sections for $t \bar{t} W$ and $t \bar{t} Z$ with their uncertainties. The obtained results are in good agreement with the SM predictions.

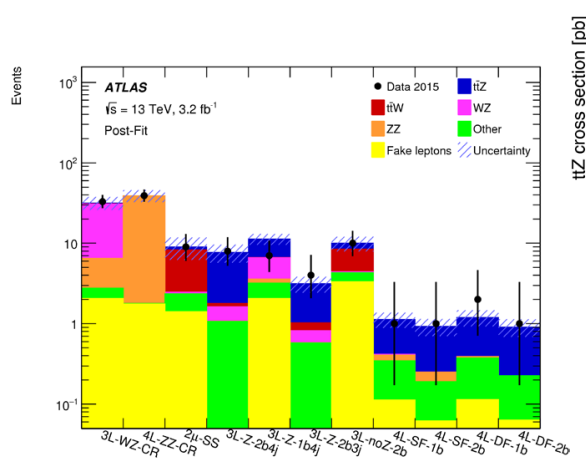

(a)

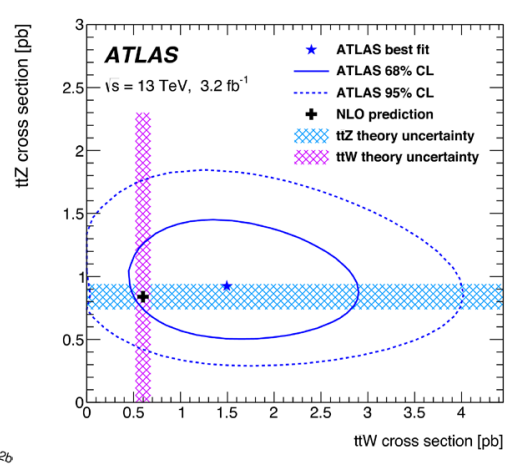

(b)

Figure 6. (a) Expected yields after the fit compared to data for the fit to extract $\sigma_{t \bar{t} Z}$ and $\sigma_{t \bar{W} W}$ in the signal regions and in the control regions used to constrain the $W Z$ and $Z Z$ backgrounds. The 'Other' background summarises all other backgrounds. The shaded band represents the total uncertainty. (b) The result of the simultaneous fit to the $t \bar{t} Z$ and $t \bar{t} W$ cross sections along with the $68 \%$ and $95 \%$ confidence level (CL) contours. The shaded areas correspond to the theoretical uncertainties in the Standard Model predictions, and include renormalisation and factorisation scale uncertainties as well as PDF uncertainties including $\alpha(s)$ variations [15].

\section{Associated production of $t \bar{t}$ and photon $(\gamma)$}

Total and differential production cross section of $t \bar{t}+\gamma$ is measured with the ATLAS detector using centre-of-mass energy $\sqrt{s}=8 \mathrm{TeV}$ [16]. The measurement focuses on semileptonic decay of the $t \bar{t}$ system. The events are separated into three categories: a) events with prompt photon originating from $t \bar{t} \gamma$ matrix element, b) events with photon from hadron decays or hadrons misidentified as photons and c) events with electrons misidentified as photons. A likelihood fit for the templates in the three categories is used to extract the total and differential cross sections of the process. The cross sections are measured within fiducial volume close to the selection requirements in the measurement and compared to theoretical NLO predictions [17]. The measured cross section is

$$
\sigma_{t \bar{t} \gamma}=139 \pm 7 \text { (stat.) } \pm 17 \text { (syst.) fb. }
$$

The measurement is limited by the uncertainty of the photon fakes modelling. Differential cross section as a function of photon $|\eta|$ and photon $p_{\mathrm{T}}$ is also measured and no significant deviation from SM prediction is observed.

\section{Top mass measurements}

The top-quark mass is one of the fundamental parameters of the SM. Loop corrections relate $m_{\text {top }}$ to the masses of Higgs and $W$ boson. Precise measurement of top-quark mass plays an important role in 
tests of SM. The stability of the electroweak vacuum depends, together with the Higgs boson mass, on a precise determination of $m_{\mathrm{top}}$.

\subsection{Dilepton channel}

The most precise measurements of $m_{\text {top }}$ exploit MC generators to create templates with different assumptions on $m_{\text {top }}$. These templates are then fitted to data and the top-quark mass is extracted.

The ATLAS collaboration measures $m_{\text {top }}$ using dilepton $t \bar{t}$ events at centre-of-mass energy $\sqrt{s}=$ $8 \mathrm{TeV}$ [18], corresponding to integrated luminosity of $20.2 \mathrm{fb}^{-1}$. Invariant mass of lepton and corresponding $b$-jet $\left(m_{\ell b}\right)$, is chosen as an observable sensitive to the top-quark mass. Templates of $m_{\ell b}$ are simulated for a number of discrete $m_{\text {top }}$ masses. From the two possible assignments of the two pairs of leptons and $b$-jets, the combination leading to the lowest average $m_{\ell b}$ is retained. Additionally, selection on transverse momenta of $\ell b$ system $\left(p_{\mathrm{T}, \ell b}>120 \mathrm{GeV}\right)$ is used to decrease to the total uncertainty. Resulting $m_{\ell b}$ templates are constructed as a Gaussian with $m_{\text {top }}$ as a free parameter and a Landau function, which give a good description of the templates within statistical uncertainty. Background is parametrized only as a Landau function. Maximisation of unbinned likelihood results in $m_{\text {top }}$

$$
m_{\text {top }}=172.99 \pm 0.41 \text { (stat.) } \pm 0.74 \text { (syst.) } \mathrm{GeV}
$$

which corresponds to relative uncertainty on the top-quark mass of $0.5 \%$. Dominant systematic uncertainties arise from jet energy reconstruction and signal modelling. The results of this measurement are combined with $\sqrt{s}=7 \mathrm{TeV}$ ATLAS collaboration measurement [19] using Best Linear Unbiased Estimate (BLUE) technique. This combination yields

$$
m_{\text {top }}=172.84 \pm 0.34 \text { (stat.) } \pm 0.61 \text { (syst.) } \mathrm{GeV} \text {, }
$$

corresponding to relative uncertainty of $0.4 \%$.

\subsection{All-hadronic channel}

The ATLAS collaboration measures top-quark mass in all-hadronic $t \bar{t}$ channel [20]. The measurement benefits from the largest branching ratio (46\%) among the possible top-quark decay channels. However, the measurement is difficult due to large multi-jet background, which can exceed the $t \bar{t}$ production by several orders of magnitude. To reduce the overwhelming multi-jet background, events are required to have at least 5 jets with tight selection of $p_{\mathrm{T}}>60 \mathrm{GeV}$. The two jets with largest $b$-tag weight are required to have $\Delta \phi\left(b_{i}, b_{j}\right)>1.5$. This requirement is very powerful in rejecting combinatorial background. Kinematics of $t \bar{t}$ system is reconstructed using $\chi^{2}$ method. The observable sensitive to $m_{\text {top }}$ is ratio of three-jet mass divided by two-jet mass $\left(R_{3 / 2}\right)$. Templates of $R_{3 / 2}$ are generated for discrete $m_{\text {top }}$ values. The templates are parametrized by combination of a Novosibirsk and a Landau function. Multi-jet background is estimated using ABCD method. Statistical uncertainty is corrected by a factor of $\sqrt{1+\rho}$ due to the correlation of two $R_{3 / 2}$ variables in an event. The measured $m_{\text {top }}$ is

$$
m_{\text {top }}=173.72 \pm 0.55 \text { (stat.) } \pm 1.01 \text { (syst.), }
$$


with relative uncertainty of $0.7 \%$. Dominant systematic uncertainties arise from jet energy reconstruction and hadronization modelling. Figure 7 shows post-fit distribution of $R_{3 / 2}$ and correlation of measured $m_{\text {top }}$ and fitted background normalization. Summary of recent ATLAS $m_{\text {top }}$ measurements is shown in Figure 8.

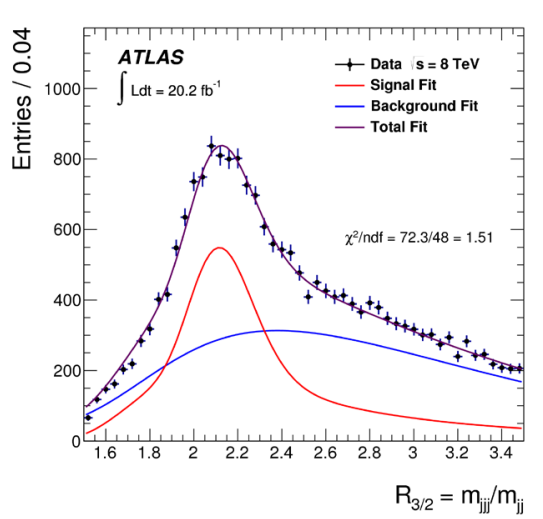

(a)

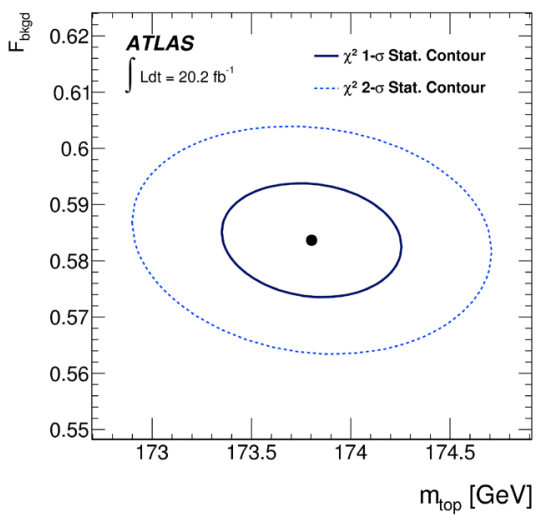

(b)

Figure 7. (a) The $R_{3 / 2}$ distribution in data with the total fit (in magenta) and its decomposition into signal (in red) and the multi-jet background (in blue). The errors shown are statistical only. (b) The ellipses corresponding to the $1-\sigma$ (solid line) and $2-\sigma$ (dashed line) statistical uncertainty. The central point in the figure indicates the values obtained for $m_{\mathrm{top}}$ on the x-axis, and the fitted background fraction, $F_{\mathrm{bkgd}}$, obtained within the fit range of the $R_{3 / 2}$ distribution on the y-axis [20].

\subsection{Top-quark pole mass measurement using $t \bar{t}+1$-jet cross-section}

Although the mass measurement from MC templates is the most precise $m_{\text {top }}$ experimental technique, it depends upon the renormalisation scheme used in the simulation and it lacks clear interpretation in well-defined theoretical picture. On the other hand, most of the theoretical calculations identify the top-quark mass with the pole mass, $m_{\mathrm{top}}^{\text {pole }}$.

Gluon radiation from $t \bar{t}$ is sensitive to $m_{\text {top }}^{\text {pole }}$. Hence, measurement of $t \bar{t}+1$-jet cross section can be exploited to extract $m_{\text {top }}^{\text {pole }}$. This measurement is more sensitive to $m_{\text {top }}^{\text {pole }}$ than inclusive cross section, which also suffers from large PDF and scale variation uncertainties. The ATLAS collaboration measures $m_{\text {top }}^{\text {pole }}$ in $t \bar{t}+1$ jet events at centre-of-mass energy $\sqrt{s}=7 \mathrm{TeV}$ [22]. The analysis focuses on semileptonic decays of $t \bar{t}$. The basic selection requires at least five jets with exactly two jets being $b$ tagged. $m_{\mathrm{top}}^{\text {pole }}$ is extracted from a measurement of normalized differential cross section $\mathcal{R}\left(m_{\mathrm{top}}^{\text {pole }}, \rho_{s}\right)$, as a function of inverse of the invariant mass of the $t \bar{t}+1$ jet system, $\rho_{s} \sim 1 / \sqrt{s_{t \bar{t}+1} \text { jet}}$. The measurement of $\mathcal{R}\left(m_{\mathrm{top}}^{\text {pole }}, \rho_{s}\right)$ in a fiducial phase-space within the detector acceptance is performed to reduce the theoretical uncertainties from extrapolation to full phase-space. The unfolding is done using Singular Value Decomposition (SVD) technique. The extracted value of $m_{\text {top }}^{\text {pole }}$ is

$$
m_{\text {top }}^{\text {pole }}=173.7 \pm 1.5 \text { (stat.) } \pm 1.4 \text { (syst.) }{ }_{-0.5}^{+1.0} \text { (theory) } \mathrm{GeV},
$$

with relative uncertainty of $1.3 \%$. 


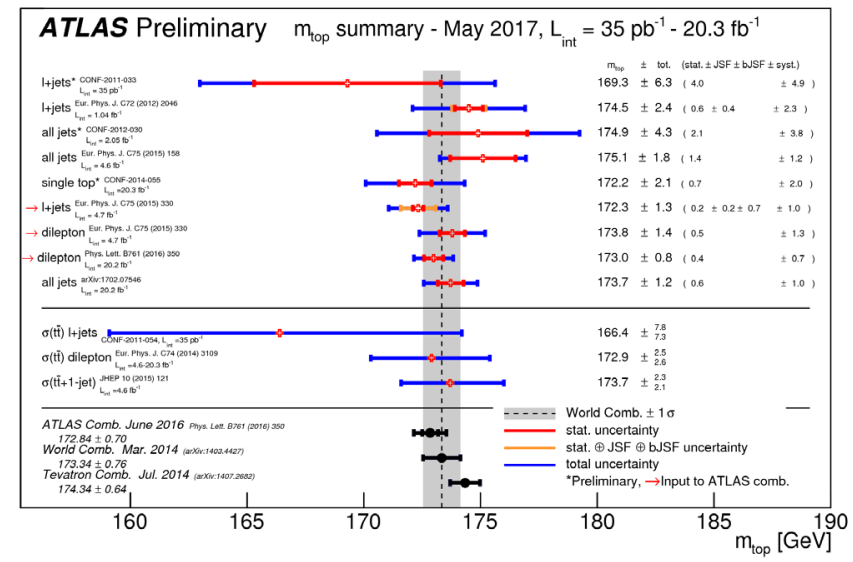

Figure 8. Summary of the ATLAS direct $m_{\text {top }}$ measurements. The figure shows the latest results, as well as previous results that are now superseeded. The results are compared with the ATLAS, Tevatron and Tevatron+LHC mtop combinations. For each measurement, the statistical uncertainty, the jet scale factor (JSF) and $b$-jet scale factor (bJSF) contributions (when applicable) as well as the sum of the remaining uncertainties are reported separately. The JSF, bJSF contributions are statistical in nature and apply to analyses performing in-situ (top-quark pair base) jet energy calibration procedures [21].

\subsection{Top-quark pole mass measurement using lepton distributions}

Very recent measurement by ATLAS collaboration exploits lepton distributions in the $e \mu$ channel of $t \bar{t}$ events at centre-of-mass energy $\sqrt{s}=8 \mathrm{TeV}$ [23]. Some of the differential cross sections of leptonic observables are sensitive to top-quark mass. Measured distributions are compared to prediction from both NLO plus parton shower event generators and fixed-order QCD calculations. The former are similar to traditional MC mass measurements but are less sensitive to hadronization modelling uncertainties as they rely purely on leptonic observables. Measurements based on fixed-order QCD calculations in a well-defined renormalization scheme correspond more directly to $m_{\text {top }}^{\text {pole }}$.

In the measurement of $m_{\text {top }}^{\text {pole }}$ from fixed-order QCD predictions, $\chi^{2}$ values are calculated for comparison of data to predictions with different $m_{\text {top }}^{\text {pole }}$ and the best-fit $m_{\text {top }}^{\text {pole }}$ is found by polynomial interpolation. The $m_{\text {top }}^{\text {pole }}$ value used in the fixed-order predictions corresponds to a well-defined renormalisation scheme, which is the pole mass scheme within the MCFM implementation. Both the QCD scale uncertainties, representing the effects of missing higher-order corrections beyond NLO, and the PDF uncertainties, are included in the $\chi^{2}$ formalism in a natural way. The measured top-quark pole mass from the combination of eight lepton distributions is

$$
\left.m_{\text {top }}^{\text {pole }}=173.2 \pm 0.9 \text { (stat. }\right) \pm 0.8 \text { (syst.) } \pm 1.2 \text { (theory) } \mathrm{GeV}
$$

which is the most precise $m_{\text {top }}^{\text {pole }}$ measurement till date with the relative uncertainty of $1.0 \%$, see also Figure 9. 


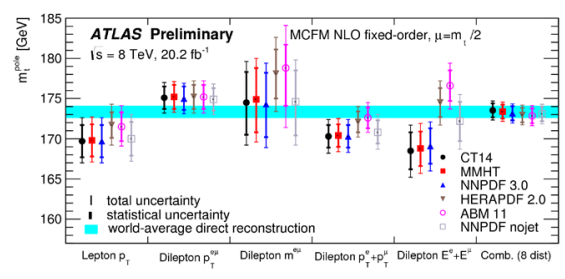

(a)

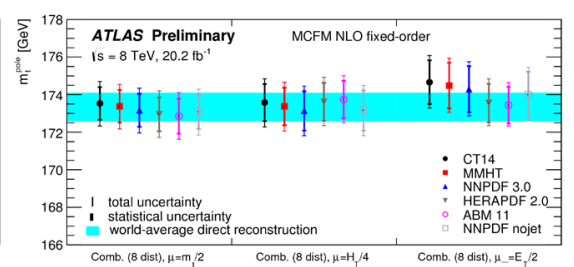

(b)

Figure 9. (a) Measurements of the top-quark mass using predictions derived from MCFM with the CT14, MMHT, NNPDF 3.0, HERAPDF 2.0, ABM 11 and NNPDF 3.0 nojet PDF sets. The central factorisation and renormalisation scales are set to $\mu_{F}=\mu_{R}=m_{\mathrm{top} / 2}$. (b) Measurements of the top-quark mass using predictions derived from MCFM with the CT14, MMHT, NNPDF 3.0, HERAPDF 2.0, ABM 11 and NNPDF 3.0 nojet PDF sets, and the central QCD factorisation and renormalisation scales $\mu_{F}$ and $\mu_{R}$ set to $m_{\mathrm{top}} / 2, H_{\mathrm{T}} / 4$ and $E_{\mathrm{T}} / 2$. The results are derived from a combined fit to all eight lepton and dilepton distributions [23].

\section{Conclusions}

This proceedings contribution reviewed some of the most recent results on the top-quark properties measurements performed by the ATLAS detector. In particular measurements of the top-quark spin correlations, $W$ helicity, top-quark decay width, top-quark mass measurements, including top-quark pole mass measurements and cross sections of associated production of $t \bar{t}$ and $W / Z$ boson or photon are reported. Additionally, searches for FCNC involving top quarks are summarized.

\section{References}

[1] ATLAS Collaboration, JINST 3, S08003 (2008)

[2] ATLAS Collaboration, JHEP 03, 113 (2017)

[3] D0 Collaboration, Phys. Rev. Lett. 80, 2063-2068 (1998)

[4] ATLAS Colaboration, JHEP 05, 061 (2015)

[5] ATLAS Collaboration, Eur. Phys. J. C 77, 264 (2017)

[6] C. Zhang and S. Willenbrock, Phys. Rev. D 83, 034006 (2011)

[7] Johannes Erdmann et. al, NIM A 748, 18-25 (2014)

[8] Andrzej Czarnecki, Jurgen G. Korner, Jan H. Piclum, Phys. Rev. D 81, 111503 (2010)

[9] J. Gao, C. S. Li and H. X. Zhu, Phys. Rev. Lett. 110, 042001 (2013)

[10] ATLAS Collaboration, ATLAS-CONF-2017-056, 2017

[11] ATLAS Collaboration, arXiv:1707.01404, 2017

[12] ATLAS Collaboration, Eur. Phys. J. C 76, 12 (2016)

[13] ATLAS Collaboration, Eur. Phys. J. C 76, 55 (2016)

[14] ATLAS Collaboration, ATLAS Top summary plots: https://atlas.web.cern.ch/Atlas/GROUPS/

PHYSICS/CombinedSummaryPlots/TOP/fcnc_summarybsm/history.html

[15] ATLAS Collaboration, Eur. Phys. J. C 77, 40 (2017)

[16] ATLAS Collaboration, arXiv:1706.03046, 2017

[17] K. Melnikov, M. Schulze and A. Scharf, Phys. Rev. D 83, 074013 (2011)

[18] ATLAS Collaboration, Physics Letters B 761, 350-371 (2016) 
[19] ATLAS Collaboration, Eur. Phys. J. C 75, 330 (2015)

[20] ATLAS Collaboration, arXiv:1702.07546, 2017

[21] ATLAS Collaboration, ATLAS Top summary plots: https://atlas.web.cern.ch/Atlas/GROUPS/

PHYSICS/CombinedSummaryPlots/TOP/mtopHistory_ATLASonly/history.html

[22] ATLAS Collaboration, JHEP 10, 121 (2015)

[23] ATLAS Collaboration, ATLAS-CONF-2017-044, 2017 